\title{
Hybrid Prior Method for Positron Emission Tomographic Image Reconstruction
}

\author{
Partha Pratim Mondal \\ Dept. of Physics, Indian Institute of \\ Science. \\ Bangalore, Karnataka, India \\ email: partha@physics.iisc.ernet.in
}

\begin{abstract}
Maximum a-posteriori (MAP) estimation has the advantage of incorporating prior knowledge in the image reconstruction procedure which makes it a superior estimation technique compared to convolution back-projection (CBP), maximum likelihood ( $M L$ ) etc. The inclusion of prior knowledge greatly improves the image quality. However, excess smoothening occurs as the MAP-iterations are continued. In biomedical imaging sharp reconstruction is oj potential use. To meet these requirements a new prior is proposed which is capable of enhancing the edges by recognizing the correlated neighbors while restoring homogeneity in the uniform regions of the reconstruction. The proposed prior serves as a post-processing technique in Bayesian domain, once an approxirnate smooth reconstruction is generated by MAP-algorithm. Simulated experiments show improved sharp reconstruction with the proposed post-processing technique.
\end{abstract}

\section{Keywords}

Bayesian Estimation, Gibbs Prior, Maximum A-posteriori Estimation, Positron Emission Tomography, Smooth Potential, Sharp Potential

\section{INTRODUCTION}

Positron emission tomography (PET) is an imaging technique for mapping physiological activities in different parts of body expecially brain. This technique comprises of doping blood with radio-compounds either by injection or inhalation. While specfic physiological process like pain, muscle movement etc. is carried out, cells concentrate these radio-compounds in different proportions inside the body. Radio-compounds being unstable emit subatomic particles which get detected in the circular detector ring placed around the object under study. Once the detector counts are registered and stored, the aim is the reconstruction of an accurate map of spatial and temporal distribution of the radiocompounds by accurate modeling of the physical processes which result in the observed detector counts.

The fact that MAP algorithm performs better than other image reconstruction algorithms such as convolution back projection, expectation maximization etc. is well established [1][2]. The MAP algorithm uses prior knowledge in the reconstruction process in addition to the likelihood knowledge for better performance. It is found that invoking homogenous priors in the reconstruction procedure re-

\author{
K. Rajan
}

Dept. of Physics, Indian Institute of Science.

Bangalore, Karnataka, India

email: rajan@physics.iisc.ernet.in

sults in the over-smoothening of the reconstructed estimates. The assumption of homogeneity breaks down near the edges of uniform regions of the reconstructed images and hence should be dealt differently. The MAP-estimation often ends up producing smoother reconstruction. This is certainly an undesirable feature which appears due to the homogenity assumed throughout the reconstruction especially at the edges. The prior proposed in this paper is capable of enhancing the edges by invoking inhomogenity in the reconstruction process without disturbing the homogenous region. The proposed method starts with an uniform estimate and a smooth MAP-estimate is generated using homogenous priors. This estimate is taken as the starting estimate for the proposed post-processing MAP-technique for edge restoration.

The paper is organized as follows. Section II provides the necessary theory behind MAP-reconstruction. The new prior is described in section III. Section IV describes the implementation issues and evaluation of the proposed algorithm, followed by conclusions in Section V.

\section{RECONSTRUCTION IN BAYESIAN FRAMEWORK}

The MAP-estimate determines that estimate $\hat{s}$ which maximizes the posterior density function i.e,

$$
\hat{s}=\arg \max _{, 0}\left[L\left({ }_{0}\right)+\log P(s)\right]
$$

where, $L\left({ }_{0}\right)$ is the log-likelihood function and $P\left({ }_{3}\right)$ is the prior distribution knowledge of the object image. . In PET, emission process is modeled as a Poisson process due to the stochastic nature of nuclear emission. The measurements of the photon counts $y_{j}, j=1 ; \ldots ;: ; M$ are also mpdeled as independent Poisson random variable with mean $i, i p_{i j}$, $J=1 ; \ldots ; ; M$, where $, i, i=1 ; \ldots ; ; N$, are mean estimates of the emission process, $p_{i j}$ is the probability of emission in pixel $\mathbf{i}$ and getting detected in the detector $j$. The loglikelihood function for PET is given by [1],

$L(s)=\sum_{j=1}^{X} i_{i=1}^{\prime}, i p_{i j}+y_{j} \log _{i=1}^{X}, i p_{i j} i \log \left(y_{j} !\right)$

The prior distribution $P($,$) for image field, is modeled as$ Gibbs distribution i.e, 


$$
P(,)=\frac{1}{7} \exp i 1^{x} x
$$

where, - and $\mathrm{Z}$ are respectively the hyper-parameter and normalizing constant of Gibbs distribution. $\mathrm{N}_{\mathbf{i}}$ represents the nearest neighbors of the pixel at site $i$, and $V(, 1 ;$,$) is$ the potential at site $\mathbf{i}$ due to its nearest neighbor,$g$.

Complicated nature of the prior distribution makes the solution of eqn.(1) very difficult. Green [3] has proposed one step late (OSL) algorithm for a recursive update of the estimates given by,

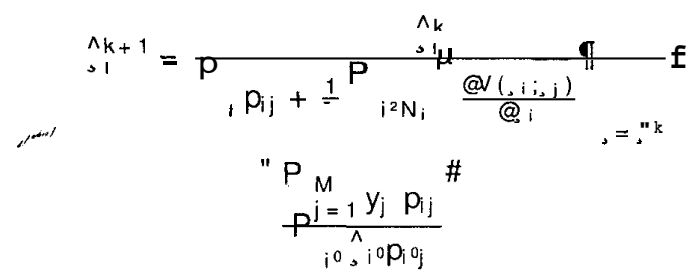

Large number of homogenous potential functions are suggested in literature [3][4][5][6]. The following potential first suggested by Geman et. al. [8] is used in this study and is given by,

$$
V_{1}(, i, j)=(, i, j, j)^{2}
$$

The general nature of these potentials is to increasingly penalize the separation between neighboring pixels irrespective of the density class, thereby introducing undesirable features like smoothening, blurring etc at the edges of the reconstructed images. To tackle this problem a new potential is proposed, which is capable of preserving the edges at the cost of few additional iterations.

\section{PROPOSED POTENTIAL}

The MAP-estimate of an image field is that estimate $\wedge$ which minimizes posterior energy. With increasing MAPiteration, Gibbs prior increases the energy of non-smooth reconstruction over smoother one. Hence, MAP-estimation proceeds toward those reconstructions, which are smoother in nature. This often results in over smoothening of edges in the reconstructed images. To deal with this difficulty, a potential is proposed in Bayesian framework that supports sharp reconstruction.

The image field is assumed as Markov random field (MRF). A MRF is said to be homogenous if Markovianity [7] is retained regardless of the relative position of the lattice site $\mathbf{i}$ in an image lattice. Hence, it is clear that if edges have to be preserved, one should restrict homogenity within uniform regions of the reconstruction. Lattice sites close to the edges but well inside a uniform region demand more contribution from neighboring sites inside the uniform region and negligible contribution from sites outside the uniform region.
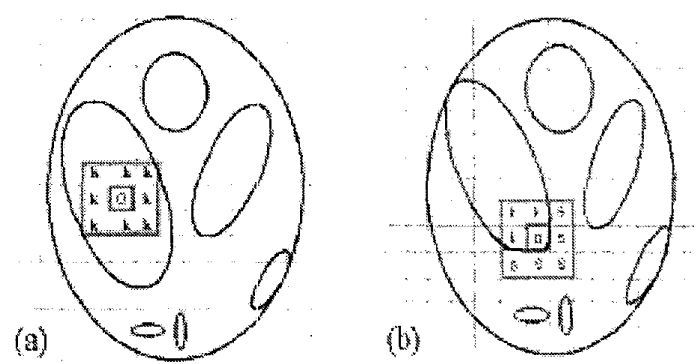

Figure 1. Schematic: diagram of Shepp-Logan Phantom on an image lattice showing, (a) site $i$ inside the uniform region (b) site $i$ at the edge but inside a uniform region.

The following potential function is found to be suitable for the preservation of edges,

$$
V_{2}(, i ;, j)=\frac{1}{j, i j, j j+ \pm}
$$

where, $\pm=0: 001$ is a tiny positive value in order to avoid singularity. So, the total potential in the neighborhood of the lattice site $i$ is given by,

$$
V_{\text {Total }}(, i, j)=x^{\prime \prime} \frac{1}{j^{2} N_{i}} \#
$$

To understand how this potential function preserves the edges, consider the following image lattice (see fig.1). Let us consider the lattice site $\mathrm{O}$ which is at the edge of two different uniform regions of the phantom image shown in Fig.1(b). Neighboring pixels are tagged by the letters $k$ and $\mathrm{S}$ respectively. Considering homogenous MRF, the potential function should increasingly penalize the difference between the pixels $\mathrm{O}$ with $\mathrm{k}$ and $\mathrm{S}$, irrespective of its density class in the image lattice. This results in the over-smoothening of the edges. Ideal prior should increasingly penalize pixels inside the uniform region i.e, pixels marked $\mathrm{k}$, and negligibly penalize pixels outside the uniform region 1.e, pixel marked $\mathrm{S}$, in order to preserve the edge information.

An edge pixel is shown in fig.1. Two different possibilities exist :

2 Pixel $O$ is inside the uniform region (see fig.1(a)). All the neighboring pixels marked $\mathrm{k}$ and $\mathrm{o}$ have similar value. So, the total potential $V_{\text {Total }}$ is large for pixels inside the uniform region. Due to large $V_{\text {T ot al }}$ it is less probable that $\mathrm{O}$ will be influenced by the neighboring pixels $\mathrm{k}$.

2 Pixel $O$ at the edge but inside the uniform region (see fig. 1(b)). Differences between pixel $\mathrm{O}$ and $\mathrm{S}$ is very large while between pixel $\mathrm{o}$ and $\mathrm{k}$ is very small. So, $V_{T \text { otal }}$ will be smaller as compared to the $V_{T \text { otal }}$ of case(1). Hence, $O$ will be greatly influenced by the neighboring pixels and it is more probable that $\mathrm{O}$ will 


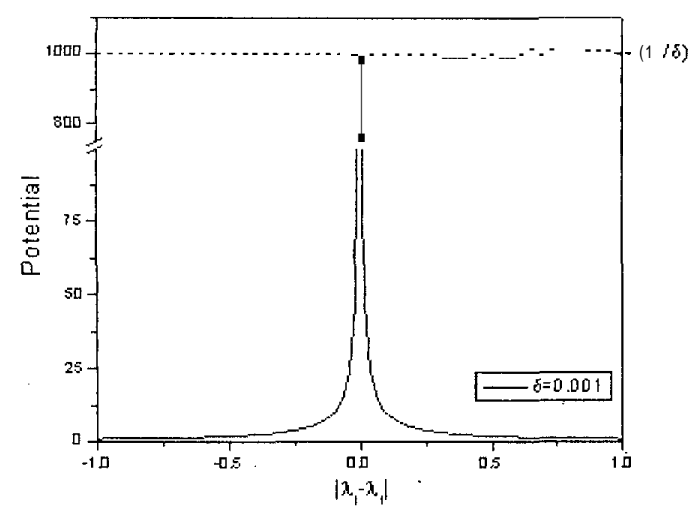

Figure 2. Plot of potential function with the pixel differences

assume value similar to $\mathrm{k}$ because the contribution from outside pixels $\mathbf{s}$ is negligible. This certainly shows the edge preserving nature of the proposed potential, the general nature of which is shown in fig.2.

Above explanation clearly suggests that the proposed potential has the ability to preserve edges without unduly effecting the homogenity in the uniform regions of the reconstruction. Once an over-smoothened MAP-reconstruction is obtained using homogenous potential (eqn.(5)) Gibbs prior, proposed potential (eqn.(6)) Gibbs prior can be employed for sharp image reconstruction. This proposed prior is intended as a post-prdcessing tool for sharp reconstruction in the Bayesian domain once the reconstruction with homogenous prior is computed. The sharpening process should be terminated well before the estimates diverge. Simulation studies show that divergence generally occurs if the preprocessing technique is continued beyond 20 iterations. One of the disadvantages of the.proposed post-processing technique is the lack of stopping criteria.

\section{SIMULATED EXPERIMENTAL RESULTS}

The proposed algorithm was implemented for a simulated PET system. The PET system consists of 64 detectors in a ring geometry and the object space is decomposed into $64 \mathrm{f} 64$ square pixels. . Before starting the reconstruction procedure, the probability matrix $\mathrm{P}=\left[\mathrm{p}_{\mathrm{ij}} ; \mathrm{i}=1 ; \ldots ; ; \mathrm{N}\right.$ and $\mathrm{j}=1 ; \ldots ; ; \mathrm{M}]$ is calculated and stored in the system. Each element of probability matrix $p_{i j}$ is the probability of an annihilation event in pixel $\mathbf{i}$, getting detected in the $\mathrm{j}^{\text {th }}$ detector and is taken as the angle seen by the center of pixel $i$ into the detector tube $j$. For simulating measurement data, a Monte Carlo procedure is used [1][9]. SheppLogan mathematical phantom with 100,000 emissions has been used for the present simulation studies. The potential shown in eqn.(5) is used for generating an approximate prior MAP-estimate.

Image reconstructed using the proposed algorithm is shown

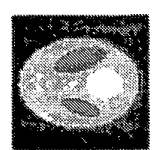

(a)

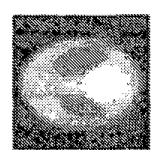

(b)

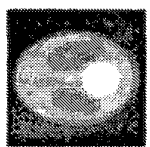

(c)

Figure 3. (a) Original Test Shepp-Logan Phantom, (b) MAP-reconstructionafter $\mathrm{k}=65$ iterations with $\mathrm{V}_{2} \mathrm{C}^{-}=$ $\left.2: 5 £ 10^{4}\right)$, (c) Hybrid Reconstruction with $V_{2}\left(^{-}=2: 5 £\right.$ $10^{7}$ ) up to $k=50$ and $V_{1}\left(^{-}=2: 5 £ 10^{7}\right)$ for additional 15 iteration

in Fig.3. It is clear that with a constant ${ }^{-}$-value, the reconstructed image is either smoother (for small - ) or noisy (for large ${ }^{-}$). In the present simulation experiment, stable reconstructions are found with ${ }^{-}$-values in the range $\left[2: 5 £ 10^{4} ; 2: 5 £ 10^{7}\right]$. Large number of simulated experimental studies shows that best MAP-reconstruction is with - $=2: 5 £ 10^{4}$. MAP-reconstruction after 65 iterations with potential $V_{1}$ and hyper-parameter ${ }^{-}=2.5 £ 10^{4}$, is shown in Fig.3(b). Experimentally, 50 iterations are found to be enough for an approximate smooth estimate using homogenous prior. Post-processing with an additional 15 iterations employing proposed potential (eqn.(6)) has produced good quality reconstruction as shown in Fig.3(c).

These results clearly demonstrate the edge preserving nature of the proposed prior and also exemplify the superiority of the proposed hybrid technique for PET image reconstruction. It is found that the estimate diverges beyond additional 20 MAP-iterations with the proposed potential. Simulated experiments shows that proposed post-processing technique cannot be carried out indefinitely since the MAP-approach with the proposed sharp potential diverges, once it exceeds 20 iterations.

\section{CONCLUSION}

In this work, a new potential function is proposed in the Bayesian image reconstruction framework. Unlike homogenous potential the proposed potential is capable of enhancing the edges keeping intact the homogeneity inside the uniform regions of the reconstruction. Smooth and sharp potential Gibbs prior are integrated in bayesian domain to produce the best of both. Simulated experiments have shown that the proposed hybrid MAP-reconstruction technique produces better quality reconstruction as compared to those realized by single prior based MAP-reconstruction technique, The results obtained using hybrid technique are very encouraging. 


\section{ACKNOWLEDGEMENTS}

The first author would like to thank Mr. Hemanth, research scholar at Indian Institute of Science for helping during the simulation experiment.

Also, he wishes thanks to Council of Scientific and Industrial research (CSIR), Government of India, for providing Junior Research fellowship. He declares that this work is partially supported by CSIR, New Delhi, India.

\section{REFERENCES}

[1] L. A. Shepp and Y. Vardi, "Maximum Likelihood Reconstruction for Emission Tomography", IEEE Trans. on Med. Img., Vol. MI-I, No.2, Oct. 1982

[2] K. Lange and R. Carson, " EM reconstruction algorithm for emission and transmission tomography", Jl. Of Computed'Assisted Tomography, 8, pp.306-316, 1984

[3] P.J. Green, "Bayesian reconstruction from emission tomography data using a modified EM algorithm", IEEE Trans. on Med. Img,, vol.9, No.1, March, 1990.

[4] J. Nuyts, D. Bequé, P. Dupont; and L. Mortelmans, "A Concave Prior Penalizing Relative Differences for Maximum-a-Posteriori Reconstruction in Emission Tomography", IEEE Trans. on Nuclear Science, Vol. 49, No. 1, pp.56-60, Feb. 2002

[5] Z. Zhou, R. M. Leahy and J. Qi, ”Approximate maximum likelihood hyperparameter estimation for Gibbs prior", IEEE Trans. on Img. proc., Vol.6, No.6, pp.844-861, June, 1997.

[6] T. Hebert and R. Leahy, " A generalized EM-algorithm for 3-D Bayesian Reconstruction from Poisson Data Using Gibbs priors ", IEEE Trans. on Med. Img., vol. 8, No.2, pp. 194-202, 1989

[7] S. Z. Li, "Markov Random Field Modeling in Computer Vision", Chapter. 1, Springer-Verlag, 1995.

[8] S. Geman and D.Geman, "Stocastic relaxation, Gibbs distribution and the Bayesian restoration of images ", IEEE Trans. Pattern Anal. Machine Intell., vol. PAMI-6, pp. 721-741, Nov., 1984 .

[9] N. Rajeevan, K. Rajgopal and G. Krishna. "Vector-Extrapolated fast maximum likelihood estimation algorithms for emission tomography", IEEE Trans. on Med. Img., Vol. 11, No.1, March 1992 